\title{
Volumetric changes of the latissimus dorsi muscle after postoperative chemotherapy in cases of immediate breast reconstruction with an extended latissimus dorsi musculocutaneous flap and implant
}

\author{
Jae Woo Lee ${ }^{1}$, Jung Yeol Seo ${ }^{1}$, Youn Joo Jung ${ }^{2}$, Ki Seok Choo ${ }^{3}$, Min Wook Kim ${ }^{1}$, Tae Seo Park ${ }^{1}$, Yong \\ Chan Bae ${ }^{1}$, Su Bong Nam ${ }^{1}$, Hyun Yul Kim ${ }^{2}$ \\ ${ }^{1}$ Plastic and Reconstructive Surgery, School of medicine, Pusan National University, Yangsan, Republic of Korea; ${ }^{2}$ Department of Surgery and \\ Research Institute for Convergence of Biomedical Science and Technology, ${ }^{3}$ Department of Radiology, Pusan National University Yangsan Hospital, \\ Yangsan, Republic of Korea \\ Contributions: (I) Conception and design: SB Nam; (II) Administrative support: HY Kim; (III) Provision of study materials or patients: HY Kim; (IV) \\ Collection and assembly of data: JW Lee; (V) Data analysis and interpretation: JW Lee; (VI) Manuscript writing: All authors; (VII) Final approval of \\ manuscript: All authors. \\ Correspondence to: Hyun Yul Kim. Department of Surgery, Pusan National University Yangsan Hospital, Beomeo-ri, Mulgeum-eup, Yangsan, \\ Gyeongsangnam-do, Korea. Email: isepa102@naver.com.
}

Background: This study aimed to evaluate the volume reduction of latissimus dorsi muscle in patients who
underwent immediate breast reconstruction using extended latissimus dorsi musculocutaneous (eLDMC) flap
with implant after skin/nipple sparing mastectomy (SSM/NSM) and postoperative chemotherapy (POCTx).
Methods: We retrospectively reviewed clinical records of 19 patients who underwent this surgery with
POCTx, and checked chest computer tomography (CT) at 7-10 days after surgery and 6-14 months after
the end of chemotherapy, from May 2015 to October 2016 . The motor nerve to latissimus dorsi muscle
was severed in all patient. One plastic surgeon intervened and the follow up period was at least 18 months.
Author obtained the area of latissimus dorsi muscle using the Picture Archiving and Communication System
(PACS) in the axial view of the chest CT and it was verified by the experienced radiologist. The statistical
test was performed $(\mathrm{P}<0.05)$.

Results: There was statistically decrease in latissimus dorsi muscle volume after the end of POCTx, range from $51.5-77.5 \%$, average volume decrease was $62 \%$, compared with latissimus dorsi muscle volume of the CT taken 7-10 days postoperatively $(\mathrm{P}<0.05)$. After a minimum follow-up period of 18 months, 14 of the 19 cases were satisfactory for both the surgeon and the patient. In 4 patients, breast volume was symmetrical when wearing bra, but the outline of the $90 \mathrm{cc}$ implant was unfavorable. One patient had a smaller size of reconstructed breast than the other.

Conclusions: The size of the implant should be determined considering that average latissimus dorsi muscle reduction is $62 \%$. Especially, the size of the implant should be chosen carefully if latissimus dorsi muscle is thick or it occupies a large portion of the entire flap.

Keywords: Breast; reconstruction; volume change

Submitted May 10, 2019. Accepted for publication Aug 14, 2019.

doi: 10.21037 /gs.2019.09.01

View this article at: http://dx.doi.org/10.21037/gs.2019.09.01 


\section{Introduction}

Breast reconstruction with an extended latissimus dorsi musculocutaneous (eLDMC) flap and implant allows skin/nipple-sparing mastectomy (SSM/NSM) and breast reconstruction to be performed simultaneously, which is advantageous for various reasons. However, the reconstructed breast volume may decrease due to postoperative atrophy of the latissimus dorsi muscle (1). Such changes may be further aggravated by intraoperative transection of the motor nerve innervating the latissimus dorsi muscle (2). This is done to prevent synkinetic movement, which can cause difficulties in achieving breast symmetry (3). Consequently, surgeons must determine the appropriate breast volume for reconstruction based on the expected atrophy of the latissimus dorsi muscle. In our previous study, we found that the average degree of volume reduction of the latissimus dorsi muscle in patients without chemotherapy or radiation therapy was approximately $54 \%$ (1). However, it is difficult to predict the degree of muscle atrophy in patients receiving postoperative chemotherapy (POCTx).

Therefore, the degree of volume reduction was assessed in patients who received POCTx following immediate breast reconstruction surgery with an eLDMC flap and implant. This study aimed to determine the degree of volume reduction of the latissimus dorsi muscle after postoperative chemotherapy.

\section{Methods}

This study was conducted among patients who received total mastectomy and immediate breast reconstruction surgery with an eLDMC flap and implant between May 2015 and October 2016. Nineteen patients who received only POCTx (i.e., without radiation therapy) and had chest computed tomography (CT) imaging at 7-10 days after surgery and 6-14 months after completion of chemotherapy were enrolled. The eLDMC flap was harvested using conventional methods, and the lumbar fat pad was not included due to the elevated risk of donor-site seroma. All implants were placed under the pectoralis major muscle via the dual plane II technique, and artificial dermal matrix was used in all patients. A sizer was used prior to implant placement, and the selected implant volumes ranged from 90 to $310 \mathrm{cc}$. Both round-type and anatomical-type implants were utilized. All reconstructive procedures were performed by a single plastic surgeon, and in all patients, the motor nerve innervating the latissimus dorsi muscle was transected. The minimum postoperative follow-up period was 18 months. The area of the latissimus dorsi muscle was calculated from the CT axial view using the Picture Archiving and Communications System (PACS) by the surgeon based on the operative method. This calculation was then verified by an experienced professor from the Department of Radiology who specialized in breast imaging. Volume was calculated from the area and was analyzed statistically, with $\mathrm{P}$ values $<0.05$ considered to indicate statistical significance.

The study was approved by the Institutional Review Board of Pusan National University Yangsan Hospital (IRB No. 05-2018-019) and performed in accordance with the principles of the Declaration of Helsinki. Written informed consents were obtained.

\section{Results}

The volume reduction of the latissimus dorsi muscle calculated on CT imaging after the completion of POCTx, in comparison with the volume at 7-10 days after surgery, ranged from $51.5 \%$ to $77.5 \%$. This change was statistically significant $(\mathrm{P}<0.05)$, and the mean volume reduction was $62.0 \%$ (Table 1). After a minimum follow-up period of 18 months, 14 of the 19 cases were satisfactory for both the surgeon and the patient; among the five unsatisfactory cases, 4 patients had a symmetric breast volume when they wore a bra but were dissatisfied with the contour of the $90 \mathrm{cc}$ implant, and in one patient, the volume of the reconstructed breast was smaller than that of the contralateral breast.

\section{Discussion}

Methods allowing the simultaneous performance of both SSM/NSM and breast reconstruction include reconstruction with a transabdominal rectus abdominis musculocutaneous (TRAM) flap or an eLDMC flap with or without an implant, as well as direct-to-implant (one- or two-stage) reconstruction (4-6). Despite the high donorsite morbidity, TRAM flaps are preferred in patients who must use autologous tissue, as this procedure does not require implants. Recently, however, implant-based breast reconstruction is receiving attention in patients who want implants or are not averse to them, due to the short operating time and reduced financial burden of implantbased procedures (5). Nonetheless, reconstructions that only use implants pose a high risk of complications, such as 
Table 1 Summary of cases

\begin{tabular}{lccc}
\hline Case & Postoperative $7-10$ day $\left(\mathrm{mm}^{3}\right)$ & After chemotherapy $6-14$ months $\left(\mathrm{mm}^{3}\right)$ & Volume decrease $(\%)$ \\
\hline 1 & $108,538.30$ & $39,281.38$ & 63.81 \\
2 & $138,054.35$ & $52,265.48$ & 62.14 \\
3 & $109,399.65$ & $43,607.75$ & 60.14 \\
4 & $110,448.70$ & $48,576.20$ & 56.02 \\
5 & $120,466.70$ & $44,718.80$ & 62.88 \\
6 & $117,764.45$ & $38,055.75$ & 67.68 \\
7 & $109,240.75$ & $42,018.45$ & 61.54 \\
8 & $94,814.85$ & $45,775.45$ & 51.72 \\
9 & $146,615.30$ & $62,340.15$ & 57.48 \\
10 & $104,784.40$ & $46,515.40$ & 55.61 \\
11 & $135,782.25$ & $39,527.30$ & 70.89 \\
12 & $134,519.65$ & $61,140.74$ & 54.55 \\
13 & $99,013.80$ & $38,588.90$ & 61.03 \\
14 & $145,893.05$ & $65,926.80$ & 54.81 \\
15 & $137,682.20$ & $31,014.30$ & 77.47 \\
16 & $96,341.80$ & $37,663.50$ & 60.91 \\
17 & $150,323.75$ & $56,751.45$ & 62.25 \\
18 & $130,353.55$ & $42,895.80$ & 67.09 \\
19 & $131,004.55$ & $38,967.98$ & 70.25 \\
Average & $122,160.11$ & $46,085.87$ & 62.01 \\
\hline & & & \\
\hline
\end{tabular}

infections and seroma. Reconstruction with an eLDMC flap and implant combines the advantages of the TRAM flap and implant-based reconstruction. However, the reconstructed breast volume may decrease due to postoperative atrophy of the latissimus dorsi muscle, which may be further aggravated by intraoperative transection of the motor nerve innervating the latissimus dorsi muscle. This transection is performed to prevent synkinetic movement (breast animation deformity), which causes difficulties in achieving breast symmetry. Additionally, objective studies on latissimus dorsi muscle atrophy are rare, especially studies focusing on the volumetric reduction of the latissimus dorsi muscle in patients receiving only POCTx.

Immediate breast reconstruction with an eLDMC flap may be performed simultaneously with total mastectomy in patients with small to medium breast volume. However, excessive flap harvesting in patients with medium to large breast volume may increase the risk of donor-site morbidity. For this reason, the simultaneous use of implants is more common in such patients. Simultaneous implant placement requires selecting an appropriate implant size in consideration of the predicted volumetric breast reduction resulting from atrophy of the latissimus dorsi muscle. A previous our study observed $54 \%$ atrophy of the latissimus dorsi muscle in patients who did not receive POCTx or radiotherapy, and such data are used as a reference point for selecting the appropriate implant size.

Many patients who undergo SSM/NSM simultaneously with breast reconstruction receive either POCTx or radiotherapy $(7,8)$; therefore, building upon previous studies, this study aimed to investigate the degree of atrophy of the latissimus dorsi muscle resulting from POCTx. Recently, it has become common for most patients to receive reconstruction using only implants or reconstruction with an eLDMC flap following partial mastectomy (breast conserving surgery). This resulted in a small sample size, as 

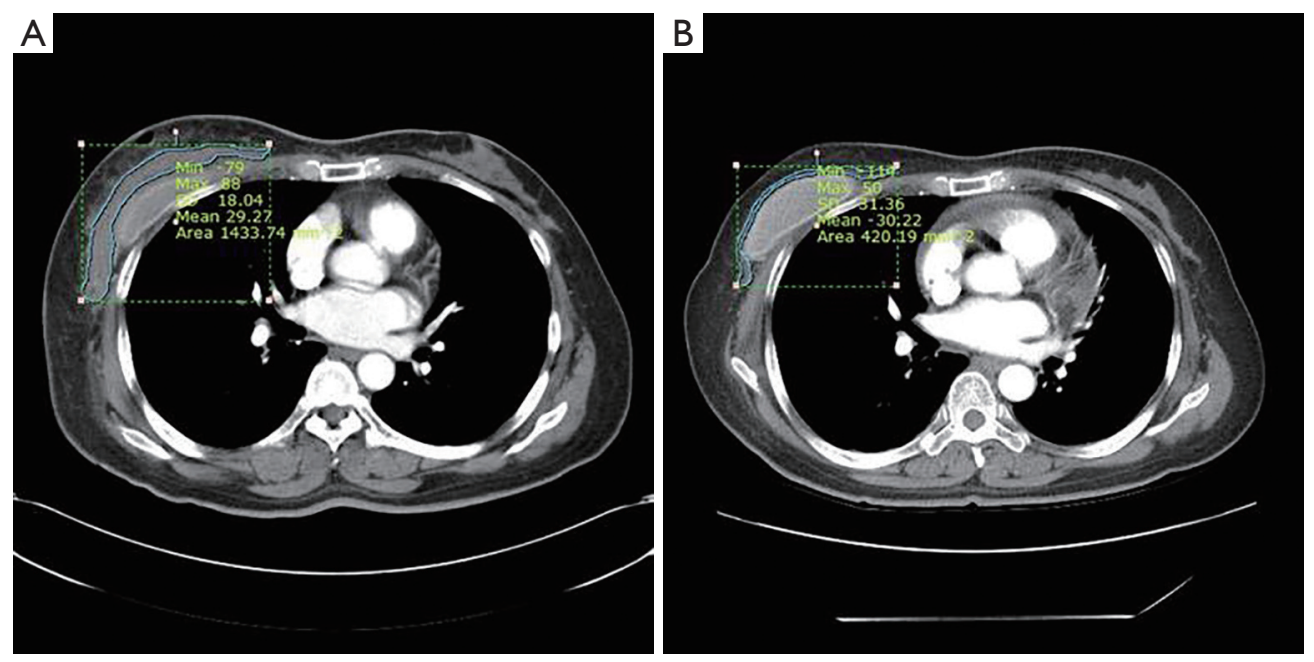

Figure 1 Chest CT-axial view: PACS system. (A) postoperative 7 days; (B) 10 months after postoperative chemotherapy. PACS, Picture Archiving and Communication System.

relatively few patients received only POCTx. Additionally, a sufficient follow-up period was required to observe any clinical outcomes, and bias was minimized by selecting patients who had same CT imaging both after surgery and after completion of chemotherapy.

All slices of images that featured the latissimus dorsi muscle in the CT axial view were measured using the PACS system of the hospital for all 19 patients, and the mean values calculated from a minimum of three measurements were used. Power testing was conducted with the assistance of the Department of Radiology (Figure 1). Although the degree of latissimus dorsi muscle atrophy according to the type of chemotherapy could not be differentiated due to the small sample size, a study is currently being conducted to yield more precise results. A mean atrophy of $62 \%$ was observed in the latissimus dorsi muscle among the 19 patients, recruited in this study, enabling a relatively precise selection of the implant size based on the resected breast mass.

Variations among patients in the thickness of the latissimus dorsi muscle and of the subcutaneous fat layer are relevant for harvesting an eLDMC flap. A higher proportion of muscle tissue in the harvested flap may result in a greater reduction in breast volume due to muscle atrophy. Thus, in patients with a thick latissimus dorsi muscle or a relatively large proportion of muscle in comparison to the subcutaneous fat layer, it is recommended to increase the implant size compared to the proposed guideline.

Bilateral breast symmetry is an important objective of immediate breast reconstruction. The size of the reconstructed breast must be quite larger than the contralateral side immediately postoperatively, after which the bilateral breast size will show closer agreement over time due to atrophy of the latissimus dorsi muscle. In this study, which had a minimum follow-up period of 18 months, 14 of the 19 patients demonstrated satisfactory outcomes for both the surgeon and the patient (Figure 2). Among the five patients with unsatisfactory results, four had a similar breast volume on both sides, but were dissatisfied of the contour of the implant due to the use of a small implant (90-120 cc); therefore, utilizing a tall height implant with less projection is expected to yield more aesthetically pleasing outcomes in patients with relatively small breasts. One subject demonstrated size asymmetry between the breasts, but did not want further corrective reconstruction.

This study has several limitations. First, there was no control group. Although we have data of patients underwent immediate breast reconstruction surgery without chemotherapy or radiation therapy, it is difficult to say that is exact control group because some conditions are different. Second, the duration of follow-up is short. As time goes on, atrophy of the latissimus dorsi muscle can progress and then the balance between both breasts can break. So, further long-term studies are needed. 

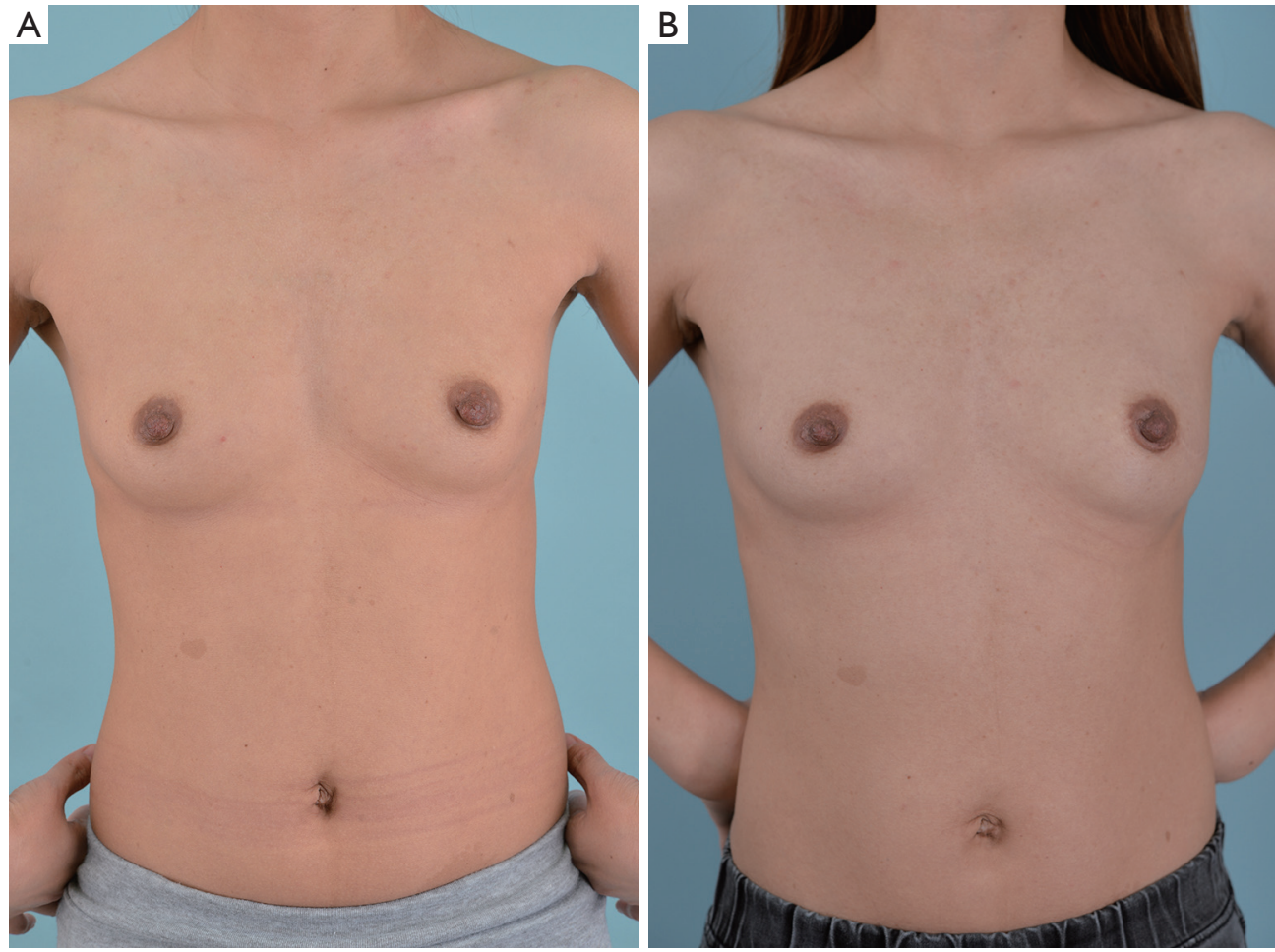

Figure 2 Photographic view. (A) Preoperative view; (B) postoperative 2 years 6 months view.

\section{Conclusions}

In patients scheduled for POCTx after SSM/NSM conducted simultaneously with immediate breast reconstruction using an eLDMC flap and implant, the reconstructed breast must be larger to anticipate the postoperative volumetric reduction of the latissimus dorsi muscle. When determing the breast implants, the latissimus dorsi volumetric reduction of about $62 \%$, as well as the shape and size of the contralateral breast, must be considered. Particular caution should be used for eLDMC flaps with a thicker latissimus dorsi muscle or with a greater proportion of muscle.

\section{Acknowledgments}

None.

\section{Footnote}

Conflicts of Interest: The authors have no conflicts of interest to declare.
Ethical Statement: The authors are accountable for all aspects of the work in ensuring that questions related to the accuracy or integrity of any part of the work are appropriately investigated and resolved. The study was approved by the Institutional Review Board of Pusan National University Yangsan Hospital (IRB No. 05-2018019) and performed in accordance with the principles of the Declaration of Helsinki. Written informed consents were obtained.

\section{References}

1. Nam SB, Oh HC, Choi JY, et al. Volumetric change of the latissimus dorsi muscle after immediate breast reconstruction with an extended latissimus dorsi musculocutaneous flap. Arch Plast Surg 2019;46:135-9.

2. Szychta $\mathrm{P}$, Butterworth M, Dixon M, et al. Breast reconstruction with the denervated latissimus dorsi musculocutaneous flap. Breast 2013;22:667-72.

3. Paolini G, Longo B, Laporta R, et al. Permanent latissimus dorsi muscle denervation in breast reconstruction. Ann Plast Surg 2013;71:639-42. 
4. Augustinho LBZ, Sabino Neto M, Veiga DF, et al. Patient satisfaction with breast reconstructionusing musculocutaneous flap from latissimus dorsiversus from rectus abdominis: a cross-sectional study. Sao Paulo Med J 2018;136:551-6.

5. Leuzzi S, Stivala A, Shaff JB, et al. Latissimus dorsi breast reconstruction with or without implants: A comparison between outcome and patient satisfaction. J Plast Reconstr Aesthet Surg 2019;72:381-93.

6. Kim H, Wiraatmadja ES, Lim SY, et al. Comparison of morbidity of donor site following pedicled muscle-sparing

Cite this article as: Lee JW, Seo JY, Jung YJ, Choo KS, Kim MW, Park TS, Bae YC, Nam SB, Kim HY. Volumetric changes of the latissimus dorsi muscle after postoperative chemotherapy in cases of immediate breast reconstruction with an extended latissimus dorsi musculocutaneous flap and implant. Gland Surg 2019;8(5):501-506. doi: 10.21037/gs.2019.09.01 latissimus dorsi flap versus extended latissimus dorsi flap breast reconstruction. J Plast Reconstr Aesthet Surg 2013;66:640-6.

7. Dutra AK, Junior JA, Fernandes ACN. Delayed breast reconstruction with transverse latissimus dorsi myocutaneous flap using Becker expander implants in patients submitted to radiotherapy: A series of cases. J Plast Reconstr Aesthet Surg 2019;72:1067-74.

8. Azzawi K, Ismail A, Earl H, et al. Influence of neoadjuvant chemotherapy on outcomes of immediate breast reconstruction. Plast Reconstr Surg 2010;126:1-11. 